\title{
Aero-Sim: A User-Centred Testbed for the Evaluation of Devices as Protection from Droplets and/or Aerosol Contaminations
}

\author{
Jessica SCHIRO $^{\mathrm{a}, \mathrm{b}}$, Alexandra DEGORRE ${ }^{\mathrm{a}, \mathrm{b}}$, Tony SANCTORUM ${ }^{\mathrm{c}}$, Thomas \\ PAGET $^{\mathrm{c}}$, Julien DEJONCKHEERE ${ }^{\mathrm{a}, \mathrm{b}}$, and Sylvia PELAYO ${ }^{\mathrm{a}, \mathrm{b}} \mathrm{l}$ \\ ${ }^{a}$ Univ. Lille, CHU Lille, ULR 2694 - METRICS : Évaluation des technologies de santé \\ et des pratiques médicales, F-59000 Lille, France \\ ${ }^{b}$ Inserm, CIC-IT 1403, F-59000 Lille, France \\ ${ }^{c}$ Univ. Lille, PRESAGE, F-59000 Lille, France
}

\begin{abstract}
Concerns over high transmission risk of SARS-CoV-2 have led to innovation and usage of an aerosol box to protect healthcare workers during airway intubation in patients with COVID-19. However, only a few studies have examined the impact of these aerosol boxes on the dispersion of droplets and aerosols, which are both thought to be significant contributors to the spread of COVID-19. In addition, to our knowledge, only a few studies have included in the concerned testbeds elements of the work context, which nevertheless have an impact on the use of the device.
\end{abstract}

Keywords. COVID-19, evaluation, usability, simulation, aerosol box.

\section{Introduction}

The reports of health care workers $(\mathrm{HCW})$ getting infected with Corona Virus in this COVID-19 pandemic are disheartening. Extensive studies and reports have found that the greatest risk of infection is during aerosol generating procedures such as intubation, bronchoscopy etc. [1]. With the growing number of cases, and increased infection of $\mathrm{HCWs}$, the need for personal protective equipment (PPE) takes prominence. The sudden surge of patients brought about an unanticipated shortage in protective equipment needed to shield healthcare workers during intubations from a highly contagious virus. Several innovations were born as an immediate requirement to tackle the problem. One of these is the aerosol box that has become popular within the anesthesia community. The aerosol box is a transparent plastic box that can be placed over a patient's head and that incorporates two circular ports through which the clinician's hands are passed to perform the airway procedure [2].

This box potentially enables physicians to intubate patients while shielding themselves from exposure to viral contamination. Nevertheless, the benefit of such device as a barrier protection in addition to the use of a standard PPE is not fully known [3]. Furthermore, its use may add another layer of complexity to performing airway intubation [4].

\footnotetext{
${ }^{1}$ Corresponding Author: Sylvia Pelayo: sylvia.pelayo@univ-lille.fr
} 
Several testbeds have been created to evaluate such type of devices [3,5-6]. To our knowledge, few, if any, simulations incorporate elements of the work context. Yet these have a definite impact on the use of the devices. It is essential that evaluations include a broader experimental setting than just a technical testbed. This will ensure a certain representativeness of the real-life use of the devices. We designed a user-centred testbed for the evaluation of devices as protection from droplets and/or aerosol contaminations.

\section{Work analysis}

\subsection{Methods}

Semi-structured interviews were conducted based on an interview grid with healthcare workers who performed interventions such as airway intubation or mechanical ventilation, as it could potentially generate aerosols of virus-contaminated respiratory secretions. Anesthetists, pulmonologists, and ENT (Ear, Nose and Throat) specialists were the most exposed to droplets and/or aerosols. The objectives of the interviews were to identify and understand the procedures in the management of a contagious patient, the equipment and specific material used, the exceptions in procedures or materials and why.

\subsection{Results}

One ENT specialist was interviewed, 2 anesthetists and 3 pulmonologists. ENT specialists, unlike other specialties, performed very few procedures on patients who were contagious or positive for COVID-19. In fact, operations were cancelled at the slightest risk. They were quickly excluded from the analyses. Anesthetists and pulmonologists had exactly the same recommendations and procedures for PPEs. PPEs (cap, goggles, or visor, FFP2 mask, gown, apron, gloves and overshoes) were available outside the rooms to avoid any contamination. Healthcare workers, therefore, dressed outside the rooms. Then, in the rooms during intubation, healthcare workers were bent over the patient's head, exposing them to droplets and/or aerosols. In an emergency, during intubation, the patient might not be fully asleep and therefore might have a cough reflex when the tube was inserted as it irritated the trachea. Extubating was also a risky procedure. Patients coughed a lot and couldn't be curarized.

In COVID-19 resuscitation wards, the number of staff per room was reduced to 2 persons. The rest of the staff had to stay in the corridor in front of the door to give the necessary material in case of need. Indeed, the resuscitation trolley was placed outside the rooms to avoid any contamination. In addition, in the COVID-19 recommendations, the use of a video-laryngoscope was requested as opposed to a laryngoscope. This avoided the need for the caregiver to position his or her head at mouth level and therefore had a slightly greater distance to the video laryngoscope screen. Finally, the whole system had to be connected before inflating the balloon because we knew that this was when cough reflexes could occur and at this moment it was already on circuit, so it projected even more.

Once the procedure had been completed and the patient's condition had been checked, the staff had to remove the PPE before leaving the room and put it in the waste bin. The removal of PPE should be done in an orderly fashion: the overshoes, the apron, the gown, the gloves, then the hand washing with gel and finally the removal of the mask and the visor and the cap by pulling forward. 


\section{User testing simulation}

\subsection{Methods}

The user testing technique was to (i) identify potential areas of contamination, (ii) test the usability of the device in an environment close to real settings, and (iii) target contexts where the risk of trans-contamination was high. Only professionals with experience in intensive care and who had been trained in the management of contagious patients (COVID or others) could be participants. Participants were randomly allocated to sequence ' $\mathrm{AB}$ ' (intubation without aerosol box and then with aerosol box) and 'BA' (intubation with aerosol box and then without aerosol box). The evaluation was conducted in a simulation lab. The Figure 1 presents the different stages of the protocol.

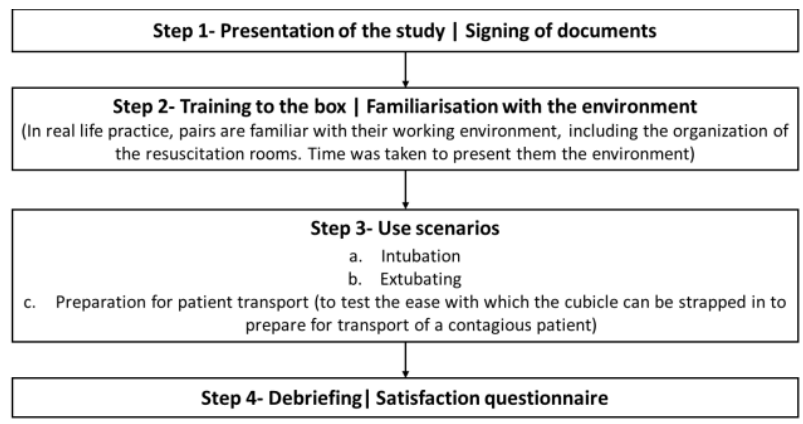

Figure 1. Different stages of the protocol.

Data collected included time for each action, the success/failure of the tasks, breach of PPEs, use errors, verbatims, and satisfaction.

\subsection{Results}

The mean time to complete one session was 2 hours. The tests were done in pairs since real intubations were performed by 2 people most of the time: the professional who intubated and an assistant (often in charge of medication, oxygen, etc.). Three different anesthetists and 4 different anesthetist nurses participated in the study as professionals who intubated. This led to a total of 7 sessions. The assistant role was acted by an anesthetist or an anesthetist nurse.
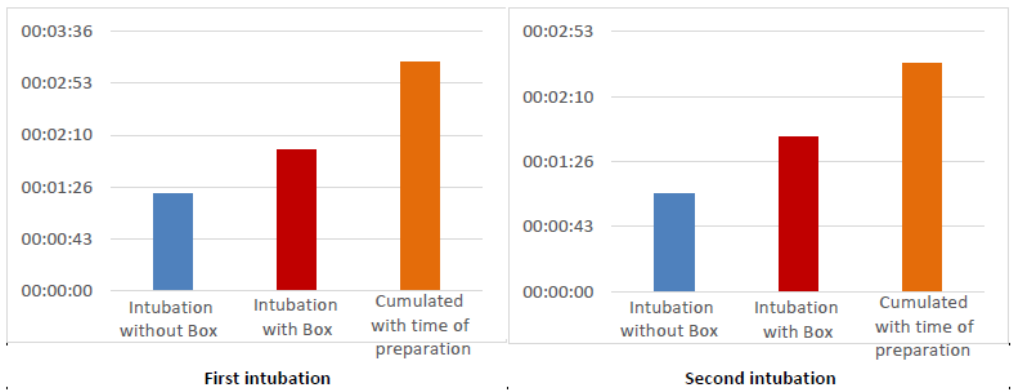

Figure 2. Mean time of intubation or intubation + preparation of box for the first and the second act. 
The mean time of intubation differed according to the condition with or without the use of the box. Intubation with the aerosol box took longer than intubation without the box (Figure 2). Moreover, if we added up the time of installation and intubation when using the protective box, the time was doubled. There was no significant difference in the first intubation (Wilcoxon test, $\mathrm{p}=0,059$ ), while a significant difference appeared in the second intubation (Wilcoxon test, $\mathrm{p}=0.03125$ ).

All participants successfully intubated the dummy with or without the box. The box was easily handled by the participants, whether it was to install it, remove it or strap it to the bed.

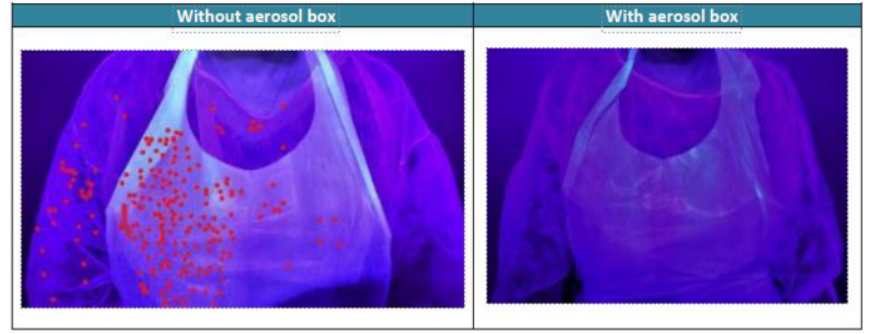

Figure 3. Example of projections observed with and without aerosol box after the intubation procedure

The aerosol box provided better protection for the participants in almost all areas of contamination (Example Figure 3), except for the hands. In fact, it did not reduce contamination since the hands were in the box during intubation. Without the aerosol box, splashes were found on the head (goggles and mask), neck, torso, arms and forearms, and hands.

Most of the participants used the box as recommended by the manufacturer, but some misuse was observed due to usability issues: diversion of the installation steps, use of the side ports for the intubation procedure instead of the one provided, passage of the material through the ports instead of through the notches provided for this purpose, extubating of the patient with the box already in the first stage of uninstallation, when removing the box after use place it on the transport trolley (which must be avoided due to risk of contamination). The aerosol box provided effective protection for operators by reducing splashes on the face and chest areas. But the observed misuses could have had an impact on the practice, both in terms of participant comfort and procedure time mainly, but to a lesser extent also in terms of risk of contamination.

Overall, the participants were satisfied. The protection is what they liked most and the security that the box provided. Its discomfort is what they disliked most, its weight "it is heavy" and its lack of space to handle the equipment.

\section{Discussion and conclusion}

Clinical simulation has gained acceptance during the last decade as a powerful qualitative method for evaluating medical devices, but also clinical information systems [7]. A simulation has to be defined to re-create characteristics of the real world. This study was conducted to design a user-centred testbed for the evaluation of devices as protection from droplets and/or aerosol contaminations, while ensuring a certain representativeness of the real-life use of the device. It has been presumed that the aerosol box protects healthcare workers against droplet and airborne contaminations during intubation. Indeed, our simulation study found that a significantly greater number of body regions 
were contaminated when performing intubation without the aerosol box compared with the aerosol box. However, our study also showed the importance of considering the work context and actual practices. Indeed, the positive results on the aerosol box efficiency were mitigated by misuses of the tool which could have ultimately increased the risk of contamination. Several participants deviated from the steps and procedure for using the box. In the simulation environment this did not prevent the accomplishment of the task but led to some adverse effects (e.g. contamination during the preparation of patient transport) which would have been dramatic in real life. Moreover, considering the entire time of the procedure (and not only the intubation time as usually in "technical" testbeds), the study also showed that intubations with the aerosol box took longer than intubation without the box when time is a critical issue in emergency situations.

The main challenge was obviously the availability of health professionals, both for first analyses to design the user-centred testbed and then for participation in the tests, especially in such a difficult period. The strong involvement of some healthcare professionals who were convinced by the innovation of the box (were even at the origin of the idea) made the project possible. But most importantly, after a first wave of COVID-19 where some new devices could be used in clinical routine without evaluation, there was a need here, from a regulatory perspective, to validate the box before a future use in real life. The design of these experimental situations is an opportunity to learn about procedures, real-life practices and facilities. This knowledge accumulates in simulation centres, and although the knowledge obviously needs to be updated regularly, it can easily be reused for other evaluations of similar devices. It could be relevant to capitalize on existing protocols with detailed versions which could be shared with the community (while paying attention to differences of contexts, cultures and healthcare systems).

\section{Funding and acknowledgement}

The authors would like to acknowledge the contributions of Dr Marc Julien of the General Hospital of Lens and Dr Grégoire Metivet of the Academic Hospital of Lille.

\section{References}

[1] Fowler RA, Guest CB, Lapinsky SE, Sibbald WJ, Louie M, Tang P, et al. Transmission of severe acute respiratory syndrome during intubation and mechanical ventilation. Am J Respir Crit Care Med. 2004;169:1198-202.

[2] Lai HY. Aerosol box, 2020. Available: https:// sites. google. com/ view/ aerosolbox/ home? authuser=0 [Accessed 31 May 2020].

[3] Noor Azhar M, Bustam A, Poh K, et al COVID-19 aerosol box as protection from droplet and aerosol contaminations in healthcare workers performing airway intubation: a randomised cross-over simulation study. Emergency Medicine Journal 2021;38:111-117.

[4] Kearsley R. Intubation boxes for managing the airway in patients with COVID-19. Anaesthesia 2020;75:969.

[5] Fried EA, Zhou G, Shah R, Shin DW, Shah A, Katz D, Burnett GW. Barrier Devices, Intubation, and Aerosol Mitigation Strategies: Personal Protective Equipment in the Time of Coronavirus Disease 2019. Anesth Analg. 2021 Jan;132(1):38-45.

[6] Begley JL, Lavery KE, Nickson CP, Brewster DJ. The aerosol box for intubation in coronavirus disease 2019 patients: an in-situ simulation crossover study. Anaesthesia. 2020 Aug;75(8):1014-1021.

[7] Jensen S, Kushniruk AW, Nøhr C. Clinical simulation: A method for development and evaluation of clinical information systems. J Biomed Inform. 2015 Apr;54:65-76. 